\title{
Acción-investigación y problemas de las minorías
}

\section{KURT LEWIN ${ }^{1}$}

El señor Baldau presenta de forma muy clara el reto que tiene la persona que está tratando de mejorar las relaciones de grupo. Aunque es capaz de dibujar un cuadro relativamente favorable de la situación en Cleveland, está ansioso por poner de manifiesto que no está en absoluto seguro de que su informe refleje algo más que lo superficial. El señor Baldau llega a enumerar importantes progresos hechos por diversos grupos minoritarios en la última década, pero no está seguro si durarán o crearán una presión lo suficientemente fuerte como para invertir la tendencia. Asimismo, duda de la efectividad de las técnicas utilizadas para mejorar las relaciones intergrupales, sin ser capaz de ofrecer sugerencias de técnicas cuya efectividad se haya probado. Sin embargo, aboga por la acción-investigación, por la investigación que ayudará al profesional. Durante el último año y medio he tenido ocasión de estar en contacto con una gran variedad de organizaciones, instituciones e individuos que han buscado ayuda en el campo de las relaciones de grupo. Entre ellos, representantes de comunidades, de sistemas escolares, de escuelas concretas, de organizaciones minoritarias de una gran variedad de características particulares y objetivos; entre ellos, también, representantes de trabajadores y de la empresa, de los departamentos de gobierno de la nación y de los estados.

Dos hechos básicos aparecen de estos contactos: existe gran cantidad de buena voluntad, de disposición para afrontar los problemas claramente y para resolverlos realmente. Si esta cantidad de buena disposición pudiera transformarse en acción organizada y eficiente, no habria peligro para las relaciones intergrupales en los Estados Unidos. Pero ahi exactamente es donde reside la dificultad. Estas personas ansiosas no ven salida. No ven salida en tres puntos: 1) ¿Cuál es la situación actual? 2) ¿Cuáles son los peligros?. Y 3) lo más importante de todo es: ¿qué vamos a hacer? Actualmente estamos realizando una entrevista entre los profesionales de las relaciones intergrupales del estado de Connecticut. Deseamos conocer su línea de pensamiento, su línea de acción y las principales dificultades con las que se encuentran. No pocas de estas personas cuyo trabajo consiste en mejorar las relaciones intergrupales piensan que quizá el mayor obstáculo a su trabajo es la falta de claridad que tienen respecto a lo que habría que hacer. ¿Cómo se puede atacar la discriminación económica y social si pensamos, no en términos generales, sino en los habitantes de esta avenida, de esa calle y de aquel barrio de una ciudad, grande o pequeña, en la que debe realizar su trabajo un determinado profesional de grupo?

1 "Action Research and Minorit Problems» Human Relations, vol. 1, part. 2, p. 34-46, 1946. Publicado por autorización de Plenum Publishing Corporation. 
Una de las consecuencias de esta falta de claridad es la inexistencia de criterios con arreglo a los cuales pueda medirse el progreso. Cuando el profesional de grupos vuelve a casa tras haber promovido una reunión llena de buenas intenciones y piensa en las personas importantes que consiguió que asistieran a ella, en todas las apelaciones conmovedoras que escuchó, en el aspecto impresionante del escenario que se montó y en la buena calidad de la comida, no tiene más remedio que sentirse lleno de entusiasmo por la atmósfera general y las palabras de alabanza que le han dirigido sus amigos. Sin embargo cuando, unos días más tarde, se entera de un nuevo caso de discriminación, se ve obligado a preguntarse si todo aquello no era más que un blanqueo de fachada y si es correcto aceptar el reconocimiento de sus amigos como una medida del progreso de su trabajo.

La inexistencia de criterios objetivos de logro tiene dos efectos graves:

1. Priva a los profesionales de las relaciones intergrupales de su deseo legítimo de satisfacción sobre una base realista. En estas circunstancias, la satisfacción o insatisfacción con el propio rendimiento se convierte en una cuestión de temperamento.

2. En un campo que carece de criterios objetivos de logro, no puede tener lugar aprendizaje alguno. Si no somos capaces de juzgar si una acción ha producido una mejora o un retroceso, si no tenemos criterios para evaluar la relación entre el esfuerzo y el logro, no hay nada que nos impida llegar a conclusiones erróneas y fomentar hábitos de trabajo incorrectos. Un requisito básico de cualquier aprendizaje es una recogida de datos y una evaluación realista. La investigación social debería ser una de las máximas prioridades del trabajo práctico de mejorar las relaciones intergrupales.

\section{CARACTER Y FUNCION DE LA INVESTIGACION PARA LA PRACTICA DE LAS RELACIONES INTERGRUPALES}

La mejor caracterización de la investigación necesaria para la práctica social es la de investigación para la gestión social o la ingeniería social. Es un tipo de acción-investigación, una investigación comparativa sobre las condiciones y los efectos de diversas formas de acción social y una investigación que conduce a la acción social. La investigación que no produce más que libros no es suficiente.

Esto no significa en modo alguno que la investigación que se necesita en este caso es menos científica o «inferior» que la que sería necesaria en el campo de los sucesos sociales. Más bien me inclino a creer que lo contrario es cierto. Instituciones interesadas en la ingeniería, como el Instituto de Tecnología de Massachussets, se han orientado cada vez más hacia lo que se conoce con el nombre de investigación aplicada. También en la ingeniería social el progreso dependerá en gran medida de la tasa con que la investigación básica en las ciencias sociales pueda desarrollar una comprensión más profunda de las leyes que gobiernan la vida social. Esta «investigación social básica» tendrá que incluir problemas matemáticos y conceptuales de análisis teóricos. Tendrá que incluir toda una amplia gama de recogida descriptiva de datos relativos a organismos sociales, grandes y pequeños. Sobre todo, tendrá que incluir experimentos de laboratorio y de campo sobre cambio social. 


\section{LA INTEGRACION DE LAS CIENCIAS SOCIALES}

Un intento de mejorar las relaciones intergrupales tiene que abordar una amplia variedad de tareas. Debe enfrentarse a problemas de actitudes y estereotipos en relación con el propio grupo y con otros grupos, a problemas del desarrollo de las actitudes y de la conducta durante la niñez y la adolescencia, a problemas de alojamiento y de cambio de la estructura legal de la comunidad; debe enfrentarse a problemas de estatus y de casta, a problemas de discriminación económica, al liderazgo político y al liderazgo de la vida comunitaria. Debe tratar el pequeño organismo social de una familia, un club o un grupo de amistad, al igual que el organismo social mayor de una escuela o un sistema escolar, el vecindario y los organismos sociales del tamaño de una comunidad, del estado, de la nación y los problemas internacionales.

Estamos comenzando a ver que es imposible atacar cualquiera de estos aspectos de las relaciones intergrupo sin considerar los otros. Esto se aplica por igual a la vertiente científica y a la vertiente práctica de la cuestión. La Psicología, la Sociología y la Antropología Cultural, cada una por su lado, han comenzado a advertir que, actuando por separado y al margen de las otras, ninguna de ellas conseguirá llegar muy lejos. Durante los últimos cinco años, primero de una forma tímida y en la actualidad muy claramente, se ha conseguido articular el deseo de un enfoque integrado. Todavía está por descubrir qué va a significar exactamente esta integración. Puede significar una amalgamación de todas las ciencias sociales en una sola ciencia social. Pero, por otra parte, puede significar simplemente la cooperación de varias ciencias en el objetivo práctico de mejorar la gestión social. Sin embargo, la próxima década presenciará, sin duda, serios intentos de un enfoque integrado en la investigación social. Soy de la opinión de que la economía tendrá que ser incluida en esta sinfonía si queremos comprender y manejar las relaciones intergrupales de manera más efectiva.

\section{DOS TIPOS DE OBJETIVOS DE INVESTIGACION}

Es importante comprender con claridad que la investigación social se ocupa de dos tipos de cuestiones bastante diferentes; a saber: el estudio de las leyes generales de la vida grupal y el diagnóstico de una situación específica.

Los problemas de las leyes generales tratan de la relación entre las condiciones posibles y los resultados posibles. Se expresan con proposiciones condicionales. El conocimiento de las leyes puede servir de guía para el logro de ciertos objetivos bajo ciertas condiciones. Para actuar correctamente no basta, sin embargo, que el ingeniero o el cirujano conozcan las leyes generales de la física o la fisiología. Tienen que conocer también el carácter específico de la situación presente. Este carácter viene determinado por una recogida científica de datos que se llama diagnóstico. En cualquier campo de acción son necesarios ambos tipos de investigación científica.

Hasta tiempos bien recientes, la recogida de datos sobre las relaciones intergrupo ha estado dominada ampliamente por las encuestas. En cierta medida, nos hemos convertido en críticos de estas encuestas de las relacio- 


\section{2}

nes intergrupales pues, aunque potencialmente son importantes, por regla general han usado métodos bastante superficiales de entrevista, desdeñando la búsqueda más profunda del tipo de entrevista utilizado por Likert que nos da cierta comprensión de las motivaciones que están detrás de los sentimientos expresados.

La segunda causa de insatisfacción es la convicción creciente de que el simple diagnóstico - y las encuestas son un tipo de diagnóstico- no basta. En las relaciones intergrupales, al igual que en otros campos de gestión social, el diagnóstico tiene que ser complementado con estudios comparativos de la eficacia de diversas técnicas de cambio.

\section{LA FUNCION Y LA POSICION DE LA INVESTIGACION DENTRO DE LA PLANIFICACION Y DE LA ACCION SOCIAL}

La ubicación adecuada dentro de la vida social de la investigación en relaciones intergrupales no es de menor importancia que su contenido. ¿Cuándo, dónde y por quién debe ser realizada la investigación social?

Dado que estamos interesados en la gestión social, examinemos con mayor detalle el proceso de planificación.

La planificación comienza generalmente con algo parecido a una idea general. Por una razón u otra parece deseable alcanzar cierto objetivo. Pero con frecuencia no está demasiado claro cómo circunscribir este objetivo y cómo alcanzarlo. El primer paso es, entonces, examinar la idea cuidadosamente a la luz de los medios disponibles. Con frecuencia se requiere recoger más datos sobre la situación. Si el primer período de planificación tiene éxito, surgen dos temas: a saber, un "plan general" sobre cómo alcanzar el objetivo y, en segundo lugar, una decisión relativa al primer paso de acción. Por lo general, esta planificación también habrá modificado algo la idea original.

El siguiente período se dedica a poner en práctica el primer paso del plan general.

En los campos altamente desarrollados de la gestión social, como por ejemplo la dirección de la empresa moderna o la ejecución de una guerra, este segundo paso va seguido por varias recogidas de datos. Pongamos un ejemplo, en el bombardeo de Alemania, una fábrica concreta se ha seleccionado como primer blanco tras la consideración cuidadosa de diversas prioridades y de las mejores formas y maneras de atacar ese blanco. Se lleva a cabo el ataque e inmediatamente se envía un avión de reconocimiento para que determine con la mayor precisión y objetividad posible la nueva situación.

Este reconocimiento o recogida de datos tiene cuatro funciones. En primer lugar, evaluaría la acción. Muestra si lo que se ha logrado supera las expectativas o queda por debajo de ellas. En segundo lugar, da a los planificadores una oportunidad para aprender, es decir, de obtener una nueva comprensión de carácter general, por ejemplo, relativa a los aspectos positivos y negativos de ciertas armas o técnicas de acción. En tercer lugar, esta recogida de datos debería servir como base para una correcta planificación del siguiente paso. Finalmente, sirve como base para la modificación del «plan general». 
El siguiente paso, de nuevo, se compone de un ciclo de planificación, ejecución y reconocimiento o recogida de datos con el propósito de evaluar los resultados del segundo paso, para preparar la base racional de la planificación dél tercer paso y para tal vez modificar de nuevo el plan general.

La gestión social racional, por tanto, procede en espiral de forma que cada paso se compone de un ciclo de planificación, acción y recogida de datos sobre el resultado de la acción.

Teniendo esto en mente, examinemos por un momento la forma en la que se manejan las relaciones intergrupales. No tengo más remedio que pensar que la persona que vuelve de celebrar con éxito una reunión bien intencionada es como el capitán de un barco que de alguna forma pensó que su barco tenía una fuerte deriva a la derecha y consiguientemente corrigió el rumbo dando un fuerte giro a la izquierda. Ciertas señales le indicaron que el timón respondía adecuadamente a dicho giro y sintiéndose feliz se fue a cenar. Pero mientras tanto, naturalmente, lo que sucede es que el barco se mueve en círculos. En el campo de las relaciones intergrupales frecuentemente la acción se basa en las observaciones hechas «dentro del barco» y en muy raras ocasiones se basa en criterios objetivos que tengan en cuenta las relaciones del movimiento del barco con el objetivo a alcanzar.

Necesitamos que el reconocimiento nos indique si avanzamos en la dirección correcta y a la velocidad a la que avanzamos. Socialmente no es suficiente que las organizaciones universitarias generen nueva comprensión científica. Será necesario establecer procedimientos de recogida de datos, ojos y orejas sociales, directamente dentro de los organismos sociales.

La idea de agencias de investigación o recogida de datos dedicadas a la mejora de las relaciones intergrupales no es nueva. Sin embargo, algunas de ellas no fueron mucho más allá de la recogida de recortes de periódicos. En los últimos años hemos presenciado desarrollos muy significativos. Hace dos años más o menos que el Congreso Judío Americano estableció la Comisión para las Interrelaciones Comunitarias. Esta es una organización de acción-investigación que se ha diseñado primordialmente para funcionar como una organización al servicio de los organismos judíos y no judios en el campo de las interrelaciones grupales. Su interés fundamental se orienta hacia el método de grupo en comparación con el método individual, por una parte, $y$, por otra, en el método de masas utilizando la radio y la prensa diaria. Estas dos últimas líneas importantes son el foco de atención de la unidad de investigación del Comité Judío Americano.

Diversos programas intentan hacer uso de nuestro sistema educativo para mejorar las relaciones intergrupales, entre ellos el Consejo Americano sobre Educación, el Estudio Universitario en las Relaciones Intergrupo en las escuelas universitarias de maestros, el Estudio de Educación Ciudadana de Detroit y, de una forma más global, la Oficina para la Educación Intercultural. Todos ellos muestran un aumento de sensibilidad hacia un procedimiento de evaluación y autoevaluación más realista, lo que es igual que decir más científico. Lo mismo se puede decir, aunque de forma diferente, de empresas específicamente dedicadas a las relaciones entre blancos y negros, como el Consejo Americano de Relaciones Raciales de Chicago, la Liga Urbana y otros. Es significativo que la Comisión Estatal contra la Discriminación del Estado de Nueva York tenga un subcomité para la coope- 


\section{4}

ración con proyectos de investigación e implicado activamente en la investigación. La creación reciente de instituciones importantes de investigación en las universidades ha ayudado también a ampliar las perspectivas de muchas de las organizaciones de acción existentes y a convencerlas de que deben confiar más en las posibilidades de usar técnicas científicas para sus propósitos.

Posiblemente no puedo intentar discutir, ni siquiera a modo de encuesta; la gran cantidad de proyectos y resultados que están surgiendo de estas empresas de investigación. Incluyen encuestas de los métodos que se han usado hasta ahora, como los que acaba de publicar Goodwin Watson; estudios del desarrollo de actitudes en niños; estudios de la relación entre actitudes intergrupales y factores tales como creencia política o la posición en el propio grupo; experimentos acerca de cómo es mejor reaccionar en caso de un ataque verbal motivado por el prejuicio; experimentos de cambio con bandas de delincuentes y con comunidades; el desarrollo de muchos tests nuevos de diagnóstico y, por último, pero no por ello menos importante, el desarrollo de teorías más precisas de cambio social. Todavía son pocos los resultados publicados de estos proyectos. Sin embargo, confío que en los próximos años presenciaremos un aumento rápido en la producción de estudios importantes y prácticos.

\section{EJEMPLO DE UN EXPERIMENTO DE CAMBIO EN PROBLEMAS DE LAS MINORIAS}

Un ejemplo puede ilustrar las potencialidades de cooperación entre los profesionales y los científicos sociales. Al principio de este año, el presidente del Comité Asesor de Relaciones Raciales del Estado de Connecticut, que es, al mismo tiempo, un miembro directivo de la Comisión Interracial del estado de Connecticut, nos pidió que dirigiéramos un seminario de trabajo con 50 asistentes sociales del campo de las relaciones intergrupales de todo el estado de Connecticut.

Surgió un proyecto en el que cooperaron tres agencias, el Comité Asesor de las Relaciones Intergrupales del Estado de Connecticut, la Comisión para las Interrelaciones Comunitarias del Congreso Judío Americano y el centro de Investigación de Dinámica de Grupos del Instituto de Tecnología de Massachusetts. El Comité Asesor del Estado se compone de miembros de la Comisión Interracial del Estado de Connecticut, de un miembro del Departamento de Estado de Educación del Estado de Connecticut y la persona que en la región del Valle de Connecticut está a cargo de la Conferencia de Cristianos y Judíos. El Estado de Connecticut parece ser el único que tiene una comisión interracial como parte de su gobierno ordinario. Parecía claro que cualquier mejora de las técnicas, que pudiese ser vinculada con este organismo estratégico central, tendría una oportunidad mucho mayor de conseguir un efecto duradero y de amplio espectro. Tras una profunda discusión de diversas posibilidades, se diseñó de forma cooperativa el siguiente experimento de cambio.

Recientes resultados de investigación han indicado que las ideologías y estereotipos que gobiernan las relaciones intergrupales no deberían considerarse como rasgos individuales del carácter, sino que están anclados en 
estándares culturales, que su estabilidad y su cambio dependen en gran medida de lo que suceda en los grupos en tanto que grupos. La experiencia con el adiestramiento en el liderazgo nos había convencido de que el escenario del seminario de trabajo es una de las herramientas más poderosas para producir una mejora de las habilidades en el manejo de las relaciones intergrupales.

Sin embargo, incluso un seminario de trabajo que obtenga un resultado positivo raramente parece poder llevar a mejoras a largo plazo en el campo de las relaciones intergrupales. El individuo que llega a su casa de un seminario de trabajo, lleno de entusiasmo y de nuevas ideas, se enfrentará de nuevo a la comunidad, encontrándose quizá él solo frente a 100.000 personas. Evidentemente, será alta la probabilidad de que su éxito no esté a la altura de su nuevo nivel de aspiración y de que pronto se deje llevar por el desánimo. Nos enfrentamos aquí a un problema de máxima importancia para cualquier cambio social, a saber, el problema de su permanencia.

Para poner a prueba ciertas hipótesis en relación con el efecto de escenarios individuales frente al de escenarios de grupo, se introdujeron ciertas variaciones en el seminario experimental de trabajo. Parte de los delegados, como es habitual, venían representando individualmente a su ciudad. Sin embargo, para un cierto número de comunidades se decidió que se trataría de tener varios delegados y, si ello resultaba posible, de desarrollar en el seminario de trabajo equipos que mantendrian las relaciones de equipo tras el seminario de trabajo. Esto ofrecería una mayor oportunidad de que permaneciera el entusiasmo y la productividad de grupo y también multiplicar el poder de los participantes a la hora de producir el cambio deseado. Un tercer grupo de delegados enviados al seminario de trabajo recibiría una determinada ayuda de expertos incluso después de haber vuelto a la comunidad.

Entre los profesores del seminario de trabajo estaban el doctor Lippitt que era el director del Proyecto, el doctor Bradford de la NEA y el doctor Benne de la Universidad de Columbia. No hay tiempo aquí de entrar en detalle en el procedimiento de adiestramiento ${ }^{1}$. Sin embargo, debería mencionar algunos puntos relativos a la investigación.

El primer paso, a la hora de llevar a cabo un diseño así, requiere una amplia recogida de datos sobre los diferentes tipos de problemas intergrupales a los que se tienen que enfrentar las diversas comunidades. Las comunidades y los equipos de profesionales de grupo en las comunidades tienen que ser seleccionados de tal forma que se puedan comprar los resultados de las tres variaciones. En otras palabras, este proyecto tenía que enfrentarse a los mismos problemas que mencionamos como típicos del proceso de planificación en general.

Las experiencias de los miembros del Consejo Asesor del Estado de la Comisión Interracial del Estado de Connecticut fueron capaces de suministrarnos rápidamente los datos suficientes para determinar las ciudades que se deberían estudiar con mayor precisión. Para evaluar el efecto del seminario de trabajo se tendría que llevar a cabo un diagnóstico antes de dicho seminario con el fin de determinar, entre otras cosas, la línea de pensamiento de los profesionales comunitarios, su principal línea de acción y los principales obstáculos a los que se enfrentaban. Algunos meses después del seminario de trabajo habría que realizar un diagnóstico similar. 


\section{6}

Para comprender porqué el seminario de trabajo produjo cambió o no, es evidentemente necesario registrar científicamente los acontecimientos esenciales durante el seminario de trabajo. Aquí es donde, en mi opinión, reside la principal dificultad de la investigación. En ningún modo sería suficiente registrar el contenido de la conferencia o del programa. La descripción de la forma de liderazgo ha de tener en cuenta la cantidad de iniciativa que han mostrado los individuos y los subgrupos, la división de los participantes en subgrupos, las fricciones dentro de cada subgrupo y entre ellos, las crisis y su resultado y, sobre todo, la pauta general de la gestión tal como va cambiando día a día. Estos aspectos a gran escala, más que cualquier otra cosa, parecen determinar lo que va a conseguir un seminario de trabajo. La tarea que los científicos sociales tienen que abordar para registrar objetivamente estos datos no es muy diferente de la del historiador. Tendremos que aprender a manejar estos períodos de tiempo y estos organismos sociales relativamente amplios sin rebajar los estándares de validez y fiabilidad a los que estamos acostumbrados en el registro psicológico de las unidades más microscópicas de acción y de los períodos de actividad en minutos o segundos.

Los métodos de registro de los acontecimientos esenciales del seminario de trabajo incluyeron una sesión de evaluación al final de cada día. Los observadores que habían asistido a las sesiones de los diversos subgrupos informaron (recurriendo a una máquina de registros) de las pautas de liderazgo que habían observado, del progreso o ausencia de progreso en el desarrollo de los grupos desde un conglomerado de individuos a un "nosotros» integrado, y así sucesivamente. Los líderes del grupo dieron su versión de las mismas sesiones y un cierto número de participantes añadieron sus comentarios.

A mí me impresionó vivamente el tremendo efecto pedagógico que sobre el proceso de adiestramiento tenían estas reuniones de evaluación que habían sido diseñadas con el propósito de registro científico. la atmósfera de objetividad, la disposición por parte de los profesores a discutir abiertamente sus errores, sin preocuparse por los riesgos a los que su posición, como tales, se exponía, parecía llevar a un aumento de la comprensión y a implantar ese espíritu de objetividad relajada que en ningún sitio es más difícil de encontrar que en el campo de las relaciones intergrupales que está cargado de emocionalidad y de actitudes rígidas, incluso, entre aquellos que a sí mismos se llaman liberales y aquellos cuyo trabajo consiste en promover las relaciones intergrupales.

Estas y similares experiencias me han convencido de que deberíamos considerar la acción, la investigación y el adiestramiento como un triángulo que debería mantenerse conjuntando por el bien de cualquiera de sus ángulos. Raramente es posible mejorar la pauta de acción sin personal de adiestramiento. De hecho, hoy, la carencia de un personal de adiestramiento competente es uno de los mayores obstáculos al progreso cuando se trata de poner a punto más experimentación. El adiestramiento de un gran número de científicos sociales que pueden manejar problemas científicos, pero que están al mismo tiempo preparados para la delicada tarea de formar equipos productivos y eficaces con los profesionales es un prerrequisito del progreso de la ciencia social así como de la gestión social de las relaciones intergrupales. 


\section{7}

Tal como puede observar durante el seminario de trabajo, los delegados de diferentes ciudades de todo el Estado de Connecticut se transformaron de una multitud de individuos, frecuentemente opuestos en su aspecto exterior y en sus intereses, en equipos cooperativos, no por la amabilidad, sino por la disposición para enfrentarse de forma realista a las dificultades, para realizar honestamente la recogida de datos y para trabajar conjuntamente para superar tales dificultades; cuando vi emerger la pauta del desempeño de roles, vi que las responsabilidades importantes pasaban lentamente, de acuerdo con el plan diseñado, de los profesores a los participantes; cuando vi, en la última sesión, cómo el Comité Asesor del Estado recibía el apoyo de los delegados para un plan que vinculaba las escuelas universitarias de maestros de todo el Estado con ciertos aspectos de las relaciones grupales dentro de las comunidades; cuando oí a los delegados y a los equipos de delegados de diversas ciudades presentar sus planes de trabajo para los seminarios a realizar en las ciudades y que otros proyectos se iban a poner en marcha de forma inmediata, no tuve más remedio que concluir que la estrecha integración de acción, adiestramiento e investigación ofrece tremendas posibilidades para el campo de las relaciones intergrupales. Me gustaría transmitirles a ustedes la misma sensación que yo he tenido.

Las relaciones intergrupales son sin duda uno de los aspectos más cruciales de la escena nacional e internacional. Hoy mejor que nunca sabemos que son, potencialmente, dinamita. La estrategia de la investigación social tiene que tener en cuenta los peligros que implican.

Podríamos distinguir entre los obstáculos y barreras externos a la ciencia social y los peligros internos de los procedimientos de investigación. Entre los primeros encontramos un grupo de personas que parece suscribir la idea de que no necesitamos más ciencia social. Entre estos admiradores del sentido común encontramos a los profesionales de todos los tipos, a los políticos y a los rectores de las universidades. Por desgracia hay un buen número de científicos físicos entre los que se oponen a una promoción vigorosa de las ciencias sociales. Parecen creer que las ciencias sociales no han producido algo de auténtico valor para la práctica de la gestión social y, por lo tanto, nunca lo harán. Creo que no hay más forma de convencer a estas personas que produciendo una mejor ciencia social.

Una segunda amenaza a la ciencia social proviene de los «grupos en el poder». Podemos encontrar a estas personas en cualquier nivel de la gestión, entre los líderes sindicales, entre los políticos, en algunas ramas del gobierno y entre los miembros del Congreso. De una manera u otra, todos ellos parecen poseídos por el temor de que no podrían hacer lo que quieren hacer si ellos, y otros, llegan a conocer los hechos. Creo que los científicos sociales deberían distinguir cuidadosamente entre los elementos legítimos e ilegítimos que están detrás de este temor. Por ejemplo, sería poco saludable que los resultados de las encuestas de Gallup determinasen automáticamente la política de lo que debería y no debería ser ley en los Estados Unidos. Tendremos que reconocer la diferencia entre la recogida de datos y el establecimiento de políticas y estudiar cuidadosamente los procedimientos por medio de los cuales la recogida de datos se podría incorporar a la maquinaria social de la legislación para producir un efecto democrático ${ }^{2}$. 
Sin embargo, es indudable que tras la hostilidad a la investigación social por parte de algunas de las personas que ocupan posiciones de poder, subyace una buena dosis de rechazo a enfrentarse a la realidad.

Un tercer tipo de ansiedad muy real por parte de los profesionales se pueden ilustrar con el siguiente ejemplo. Los miembros de los consejos comunitarios a los que tuve ocasión de informar de los resultados de la investigación sobre las relaciones intergrupales reaccionaron expresando el sentimiento de que los científicos sociales de la universidad o de cualquier rama de investigación de alguna organización nacional estarían, más tarde o más temprano, en posición de decir a los profesionales de las comunidades locales de todos los Estados exactamente lo que tienen y no tienen que hacer.

Evidentemente están pensando en una «tecnocracia» de la ciencia social. Este miedo parece un malentendido muy común basado en el término «ley». Los profesionales comunitarios no han caido en la cuenta de que la legalidad en las ciencias tanto físicas como sociales significa una relación condicional, una vinculación entre condiciones hipotéticas y efectos hipotéticos. Estas leyes no dicen qué condiciones existen localmente, en un lugar y en un momento dados. En otras palabras, las leyes no hacen el trabajo de diagnóstico que ha de hacerse localmente. Ni tampoco prescriben la estrategia de cambio. En la gestión social, al igual que en la medicina, el profesional tendrá habitualmente la posibilidad de elegir entre diversos métodos de tratamiento y necesitará tener tanta habilidad e ingenio como el médico para hacer tanto el diagnóstico como el tratamiento.

Parece crucial para el progreso de la ciencia social que el profesional llegue a comprender que a través de las ciencias sociales y sólo a través de ellas puede esperar alcanzar el poder necesario para hacer un buen trabajo. Por desgracia en las leyes sociales y en la investigación social no hay nada que obligue al profesional a elegir lo bueno. La ciencia da más libertad y poder a ambos, al médico y al asesino, a la democracia y al fascismo. El científico social debería reconocer su responsabilidad también en este sentido.

\section{INVESTIGACION SOBRE MAYORIAS Y MINORIAS}

No ha sido intención de este trabajo discutir resultados detallados de la investigación social en las relaciones intergrupales. Sin embargo, me veo obligado a mencionar dos puntos que ilustran, a mi juicio, aspectos básicos.

Las relaciones intergrupales son un asunto bidireccional. Ello significa que para mejorar las relaciones entre grupos han de estudiarse los dos grupos interactuantes.

En los últimos años hemos comenzado a advertir que los problemas etiquetados como de la minoría son en realidad problemas de la mayoría, que el problema de los negros es el problema de los blancos, que el problema judío es el problema de los no judíos y así sucesivamente. También es cierto, por supuesto, que las relaciones intergrupales no se pueden resolver sin alterar ciertos aspectos de la conducta y los sentimientos del grupo minoritario. Uno de los más firmes obstáculos para mejorarlas es la notoria ausencia de confianza y autoestima de los grupos minoritarios. Los grupos 
minoritarios tienden a aceptar el juicio implícito de aquellos que tienen mayor estatus, incluso en aquellas ocasiones en que el juicio va dirigido contra ellos mismos. Hay muchas fuerzas que tienden a desarrollar en los niños, en los adolescentes y en los adultos de las minorías un profundo antagonismo contra su propio grupo. Un grado excesivo de sumisión, de emo- . cionalidad culposa y otras causas y formas de conducta ineficaz siguen a tal antagonismo. Ningún individuo o grupo que esté en desacuerdo consigo mismo puede vivir normalmente o en armonía con otros grupos.

Debería quedar claro para el científico social que no cabe afrontar este problema proporcionando la autoestima suficiente a los miembros del grupo minoritario en tanto que individuos. La discriminación que experimentan estos individuos no va dirigida contra ellos en tanto que individuos sino en tanto que miembros de un grupo y sólo elevando su autoestima como miembros del grupo a un nivel normal se puede proporcionar el remedio.

Muchos blancos del sur parecen advertir que un prerrequisito del progreso es el aumento de la autoestima del negro del sur. Por otra parte, la idea de un programa positivo de incremento de las lealtades al grupo parece paradójico para muchos liberales. Parece que nos hemos acostumbrado a vincular la cuestión de la lealtad del grupo y de la autoestima del grupo con el patrioterismo.

A mi juicio, la solución sólo se puede conseguir a través de un desarrollo que eleve el nivel general de la estima y de la lealtad grupales, que en sí mismos son fenómenos perfectamente naturales y necesarios, al mismo nivel para todos los grupos de la sociedad. Esto significa que hay que realizar todos los esfuerzos posibles para rebajar la inflada autoestima de los ególatras. Estos deberían aprender la oración de la comedia musical Oklahoma. "Dios mío, ayúdame a comprender que no soy mejor que los otros hombres." Sin embargo, es esencial aprender la segunda parte de esta oración que dice algo así como "pero que soy tan bueno como cualquiera de ellos». De las experiencias que hasta ahora se han llevado a cabo me atrevería a afirmar que el aumentar la autoestima de los grupos minoritarios es uno de los medios más estratégicos para la mejora de las relaciones intergrupales.

El último punto que me gustaría mencionar se refiere a la relación entre la escena local, nacional e internacional. Nadie que trabaje en las relaciones intergrupales puede cerrar sus ojos al hecho de que en la actualidad vivimos en un solo mundo. Da igual que políticamente llegue a ser uno solo o dos mundos, pero de lo que no hay duda es de que, por lo que respecta a la interdependencia de los acontecimientos, estamos viviendo en un solo mundo. Tanto si pensamos en los católicos o en los judíos, en los griegos o en los negros, todos los grupos de los Estados Unidos se ven afectados profundamente por los acontecimientos que ocurren en otros lugares del globo. Las relaciones intergrupales en este país estarán moldeadas en gran medida por los acontecimientos de la escena internacional y particularmente por el destino de los pueblos coloniales. Será crucial el que este país siga o no la política que Raymond Kennedy ha denominado política internacional "Jim Crow" de los imperios coloniales. ¿Estaremos dispuestos a abandonar la política seguida en Filipinas y a volver, en las relaciones con los países que dependen de los Estados Unidos, a aquella política de explotación que ha hecho del imperialismo colonial la institución más odia- 
da en todo el mundo? ¿O seguiremos la política que John Collier ha desarrollado en relación con los indios americanos y que el Instituto de Asuntos Etnicos está proponiendo para los países que dependen de Estados Unidos? Esta es una pauta que lleva gradualmente a la independencia, la igualdad y la cooperación. Cualquiera que sea el efecto de una política de explotación permanente en la escena internacional, no se podrá impedir que tenga un profundo efecto en la situación que se viva dentro de los Estados Unidos. La política "Jim Crow" en la escena internacional perjudicará de forma muy notable el progreso de las relaciones intergrupales dentro de los Estados Unidos y muy probablemente pondrá en peligro todos los aspectos de la democracia.

El desarrollo de las relaciones intergrupales está indudablemente llena de peligros y el desarrollo de la ciencia social en este campo se enfrenta a muchas dificultades. Sin embargo, el cuadro general que he dibujado, el progreso de la investigación y muy especialmente el que la organización de la investigación social ha realizado durante los últimos años, me hace pensar que hemos aprendido mucho. Un gran esfuerzo de la investigación social de las relaciones intergrupales indudablemente podría tener un efecto duradero en la historia de este país.

Sin embargo, es igualmente claro que este trabajo exige de los científicos sociales una extraordinaria dosis de coraje. Necesita el coraje tal como lo define Platón. Necesita lo mejor de lo que los mejores de entre nosotros pueden dar, y la ayuda de todos.

1 Véase el resumen del procedimiento de adiestramiento en «Training Community Leadership toward more effective group living" de Palmer Howard y Ronald Lippitt. Adult $E d u$ cation Bulletin, August, 1946.

2 Véase "Public Opinion Polls and democratic Leadership" de Dorwin Cartwright, Journal of Social Issues, vol. 2, n. ${ }^{\circ}$, mayo 1946, pp. 23-32. 